\title{
Construction and Application of Cloud Data Center in University
}

\author{
Hong Chai \\ Institute of Railway Technology, \\ Lanzhou Jiaotong University, \\ Lanzhou, China \\ chaihong@mail.lzjtu.cn
}

\author{
Jiuyuan Huo \\ School of Electronic and Information Engineering, \\ Lanzhou Jiaotong University, \\ Lanzhou, China \\ huojy@1zb.ac.cn
}

\begin{abstract}
Construction of university Data Center is the requirement of higher education to adapt to information society, is also an important part of the construction of universities. At present, due to the informationization construction of universities lacking of unified planning and deployment, as well as the research and application of the Data Center is not enough in-depth, the needs of current management and service works could not be meet that result in delaying the informationization development. Taking the construction of Cloud Data Center to realize wisdom campus as the goal, this paper puts forward a set of construction plan of Data Center based on Cloud Computing that suitable for the application characteristics of universities, and implemented the plan in the Cloud Data Center project in Lanzhou Jiaotong University. Implementation of Cloud Data Center project in university could create a reliable platform for construction, circulation and sharing of information resources, and have obvious advantages in promoting ability of informationization integration application, management efficiency of information system, and quality of Data Center operation and reducing operation cost.
\end{abstract}

Keywords- Cloud computing; Data Center; Virtualization; IaaS; PaaS; SaaS

\section{INTRODUCTION}

In recent years, with the advancedments of information systems in universities, information technologies that based on Digital Campus have developed very quickly in higher education [1], universities have made great progress in the overoall application system construction, and a variety of information technology applications are promoting the changing in teaching, research, learning, lifestyle of teachers and students. Digital Campus puts forward higher requirements for University's informationization construction, but also provides a foundation for Data Center in the campus. University Data Center is an important part of Digital Campus, is also a major infrastructure for hosting the Digital Campus platform and application information systems to ensure that the various application systems in Digital Campus could operate in stable and efficient manner.

As the platform of normal operations of critical businesses such as teaching and daily management and the cornerstone for further development, Data Center of Lanzhou Jiaotong University campus has put forward more higher requierments for data integrity for information, reliability of business operations, availability of required network system of key businesses and core applications. And as information technology gradually penetrated into the teaching, research, office and other core businesses in university, the number of all kinds of information systems and data stored are more and more increasing, the scale of Data Center is also growing. The traditional Data Center has many common problems that still can not been resolved such as poor business continuity and flexibility, long service deployment cycle, management and maintenance for high costs and high load applications (e.g. the concentration course selection in each semester) and other issues, thus, it has been unable to meet the construction needs of the current digital campus. More and more colleges and universities have recognized the necessity of centralization of data, IT infrastructures, and running services. Thus, as the infrastructure in university, how to plan, build, operate and maintain reasonably the Data Center has become the top priority of informationization construction in universities [2].

Cloud Computing is an emerging method of shared infrastructure, it could connect the huge pool of systems together to provide a variety of IT services, and complete efficient computing and information access through Data Centers and servers which connected the Internet to make computing ability could be provided for customers like power supply [3]. Based on Cloud Computing technologies, a unified Cloud Computing service system can be deployed in campus to integrate the overall teaching and research resources on campus, and reengineer the school management processes to reduce overall information technology investment and greatly improve the resource utilization. It also could provide easy access to a variety of information services for users to enhance customer satisfaction, and the centralized management could reduce maintenance costs to free each user largely from the tedious computer maintenance.

In this paper, we combined the demands of information services on campus and characteristics of University's informationization development to build a Cloud Data Center that comply with the needs of smart campus building. Through the in-depth analysis of key technologies of Cloud Data Center in university, a Data Center construction program based on Cloud Computing that suitable the application characteristics was presented, and an unified cloud computing service was built on the campus to achieve 
a unified and transparent computer services for various departments.

\section{RELATED TECHNOLOGIES}

\section{A. Cloud Computing}

Cloud Computing [4] is a model for Internet-based services adding, using and delivery, and usually is involving to provide a dynamic, scalable and often virtualized resources via the Internet. The object of Cloud Computing is to integrate a lot of relatively low cost computing entities into a computing entity that havs the powerful calculation capabilitie through the network, and distribute this powerful computing power capacity to end-users by advanced business model. Cloud Computing is both a technology and a service that taking network as its center and service as its providing way to achieve transparency and pooling of resources. It has many advantages such as high scalability and high reliability, virtualization, large-scale, versatile, ondemand service, cheap, and son on.

SaaS, PaaS and IaaS are the three basic modes of cloud computing service model for users to get the services from Cloud Computing platform [5]. SaaS (Software as a Service) is a set of applications that providng on-demand service by way of multi-tenancy. PaaS (Platform as a Service) is to take the platform (middleware) as a service to provide a shared middleware system for users to deploy and run applications in the Cloud. Users do not need to download and install softwares. IaaS (Infrastructure as a Service) is to integrate logts of server hardwares through Cloud techonogies to form a virtual resource pool of high-performance, unified management, demand assignment, rental services to provide users a virtualized infrastructure.

\section{B. Virtualization}

One of the most important and basic technology is the virtualization technology in Cloud Computing. On the computer, virtualization is a creation of virtual version rather than the actual version, such as hardware platforms, operating systems, middlewares, storage devices or network resources. The goal of virtualization is to centralize administrative tasks, and improving scalability and workloads [6].

Virtualization technology could improve the dynamic scalability of system, reusability and management convenience of devices, the utilization of hardwares and softwares to solve a single hardware shortage, and facilitate deployment, migration, and disaster recovery of system architecture to integrate, dynamic expand and reuse resources, realize isolation of scope. Computer virtualization includes software virtualization, platform virtualization and infrastructure virtualization. Virtualization technology is one of the major driving forces to promote developments of Cloud Computing, Data Center. It can improve hardwares' utilization and flexibility, help to reduce the number of servers in the Data Center, and optimize the allocation of resources and simplify management. Using virtualization and Cloud Computing technologies to achieve a dynamic IT infrastructure environment to further improve the existing structure and management has been recognized and accepted by many universities.

\section{Data Center}

Development of Data Center has experienced four stages [7]. The first stage is Physical Data Center which is the traditional construction mode of Data Center. All systems including server systems, storage systems and network systems and associated software systems are all exist in physical form. System operation relies on independent physical device. It has the shortcomings such as low resource utilization, difficulties for deployment and management and others.

The second stage is Virtualized Data Center. Data Center began to use virtualization technology to 'split' the physical devices, so that a single physical device can be virtualized into multiple independent virtual devices to transparently provide services for the upper application systems. Virtualization includes server virtualization, storage virtualization, network virtualization and even platform virtualization. Virtualization technology could fully improve resource utilization, save investment to the system, reduce the energy consumption of Data Center operation to improve the operation flexibility and reduce operating costs.

The third stage is the Infrastructure Cloud. With Cloud Computing technology maturing, Data Centers began to use Cloud Computing techniques to build various private Infrastructure Cloud. Through unified management of scattered virtualized individuals to form various independent infrastructure resource pools, which are unified distribution, unified management and operation to achieve the most primitive construction of Infrastructure Cloud. It greatly improves resources utilization, flexibility degree, system automatic deployment, and the overall operating performance of Data Centers.

The fourth stage is the Hybrid Cloud. Through a variety of cloud computing technology, based on the construction of private Infrastructure Cloud, Hybrid Clouds on campus were constructed in the manner of unified planning and deployment, unified management for a variety of groups in various fields and various purposes such as Highperformance Computing Cloud, application Cloud, shared resources Cloud. Now, the Hybrid Cloud is the current development direction and main goal of Cloud Computing construction of universities' informationization technology $[8,9,10]$.

\section{SyStEM ARChITECTURE OF UNIVERSITY DATA CENTER BASED ON ClOUd COMPUTING}

Construction of Data Center on campus is a long and arduous task. To achieve the construction goals, a practical design must be developed. As shown in Fig. 1, the system architecture of Data Center in Lanzhou Jiaotong University is divided into the five major parts. Various parts complements each other to achieve the integrity and unity of the whole system. 


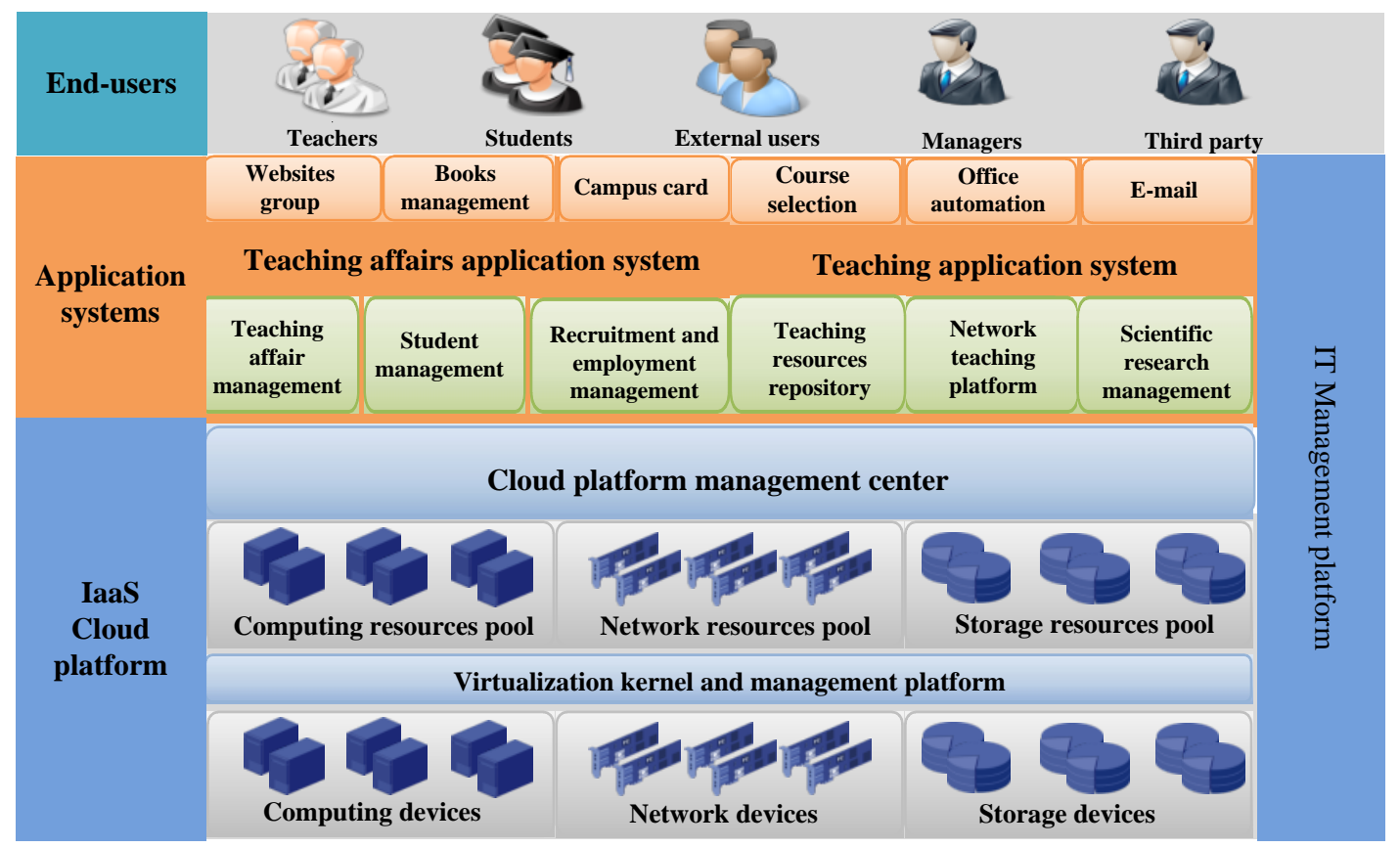

Figure 1. System structure of Lanzhou Jiaotong University Cloud Data Center

\section{A. Infrastructure and Resources}

Infrastructure provides basic support operating environment for the entire Data Center, includes network environment, server host, storage and other infrastructure hardwares. Resources are constituted by the database systems, application servers, directory servers, and other information and data resources.

- Cloud Network

Through the network virtualization, the network devices in Data Center can be used in a manner that is unrelated to physical location, physical presence and physical state. Network virtualization is to realize network resource reuse, reduce management and maintenance complexity, improve network utilization and ensure consistency of key global service strategy. It is also the foundation for the automated transfer of resources and configuration.

- Computing resource virtualization

Virtualization of computing resources are realized through virtualization software on servers. The virtual machines are taken as the main computing resources to provide services for customers, and the resources allocation in Cloud resource pools could be flexibilly deployed based on the actual business needs. The most widely used and the most significant representatives of the server virtualization software in universities are VMware vSphere, Hyper-V and XenServer. The vSphere virtualization suite is currently the only available software for production environment, and has obvious advantages to other similar products.

- storage resource virtualization

Storage system is the core foundation of the Data Center, and its performance and quality have a significant impact on the overall situation, thus how to ensure that the storage system can continue to improve, develop and maintain the system 's usability and the advanced technology is a key issue must be considered in the entire Data Center building program. Virtualized storage is taken as the primary storage resources to provide a distributed, highly secure, highly scalable storage capacity services for customers to meet the needs of high-speed data processing, mass storage, mass analysis. The basic principle of storage system construction in Cloud Data Center is to utilize virtualization technology and advanced large-capacity storage devices to centralized, intelligent manage all storage devices to form storage resource pools.

\section{B. Cloud Computing management platform}

Cloud computing management platform is responsible for managements of the entire data center resources, and the management of each resource pools and delivery of workload platform. Cloud management platform should be able to provide a unified cloud management interface to different resource pools to achieve centralized managements of each resource pools, including resource management, monitoring, configuration, diagnostics, fault detection, auditing and statistical data collection, etc. The platform should have a good scalability of cross-platform and functionality, system users can achieve self-application and management through a Web interface, and system administrators are no longer need to manually generate and configure virtual machines requested by the user, but by the management platform automatically.

\section{University Cloud application systems}

University Cloud application systems are all kinds of concrete implementations a variety of applications that 
providing for various roles. For examples, the human resources management system, educational management system, office automation system, mail systems and other public services system.

\section{Campus Cloud services}

The Cloud Data Center should provide users with various forms of Cloud services, such as (1) Infrastructure Cloud Service that is to establish a unified Cloud infrastructure by virtualizing the centralized server systems and storage systems, and provide services to users through the IaaS service model. Through virtualization suite's functions of dynamic migration and allocation of resources dynamically to ensure high availability, performance requirements and great flexibility. (2) public platform Cloud services which includes middlewares, Web servers, and database services. Through the software virtualization technology, building a unified common platform available to users through PaaS way. Users only need to apply virtual services, Web virtual hosts and database's virtual space from management center middleware, then deploy their applications to save investment, costs of operation and maintenance.

\section{E. Data Center supporting system}

\section{- Information security system}

Campus Data Center should not only ensure the safe operation of various applications, but also ensure the security of data, so the system architecture should fully consider the overall safety of the campus Data Center from physical, networks, systems, information and management to establish the security supporting system to protect safe and reliable operation of information systems on campus.

\section{- Operation and Maintenance system}

Operation and maintenance system includes system monitoring, system management, maintenance services and so on. Virtualization platform itself has capability of load balancing for computing resources, storage resources within the Cloud platform, and persistently monitors CPUs, distribution and use of memory resources of all cluster hosts and virtual machines, and perform the corresponding operation of virtual machine migration or provide migration recommendations according to running situation, thereby to maintain load balancing for computing resources.

\section{CONSTRUCTION AND APPLICATION}

According to the idea of building and construction method described in this paper, and combing Cloud Computing technology to the basis of our university's Data Center to complete the overall design of Cloud Data Centers. The construction progress of Cloud Data Center is divided into five stages: (1) The first stage: Campus data centralization, that is achiving the centralized management and maintenance of core data resources on campus, such as electronic enrollment, electronic courseware, training resources, multimedia learning resources, Email system, campus card system, library system, educational system, student management system, BBS, human management systems. (2) The second stage: IT resource virtualization that is to integrate distributed IT resources from various faculties to form resource pools through virtualization, implement security solutions to match the needs of virtualization, and avoid the effects to business continuity caused by virtualization. (3) The third stage: Management and operation of campus Cloud that is to develop processes for the Cloud resources using of faculty, personal, to rationally plan resources, and to support self-application and using cloud resources for teachers and. (4) The fourth stage: Openness and integration that is to integrate heterogeneous data resources based on Openstack technologies, to connect each virtual resources with Digital Campus using API, and to achieve key, particular business migrating to campus Cloud. (5) The fifth stage: Implementation of demonstration applications that is to implement typical applications oriented for faculty, teachers and students, train users for familiar with the using method of Cloud service, and gradually expand the coverage of campus Cloud to the existing Digital Campus's businesses.

According to the idea of building and construction method described in this paper, and combing Cloud Computing technology to the basis of our university's Data Center to complete the overall design of Cloud Data Centers. The schematic diagram of Lanzhou Jiaotong University Cloud Data Cente was shown in Fig.2. 


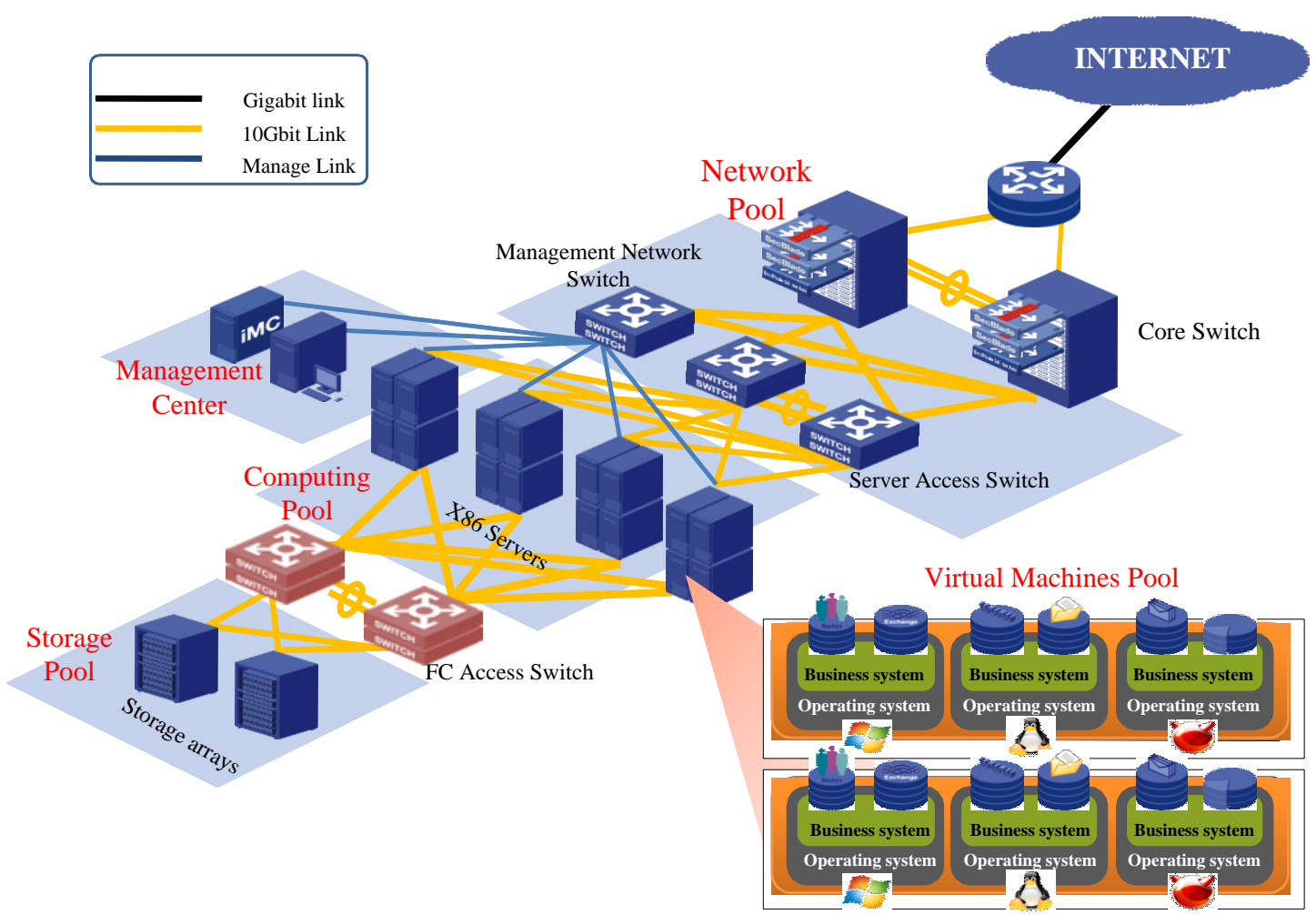

Figure 2. Schematic diagram of Lanzhou Jiaotong University Cloud Data Center

\section{CONCLUSIONS}

Data Center system construction of our university is being transformed from simple hardware to platform, from simply support campus information systems to support innovation of students and scientific transformation support of teachers to establish a reliable support platform for construction of information resources, flow and sharing of information on campus, and provides advanced tools and instruments to train highly qualified personnel of teaching, research and management. Based on the experiences and lessons of Lanzhou Jiaotong University in the Data Center construction, a set of construction plan of Data Center based on Cloud Computing was proposed to meet the needs of Digital Campus. The implementation of Cloud Data Center has significantly reduced the operating costs of information technology on campus, and effectively improves the construction quality of Cloud Data Center. But the construction of Cloud Data Center is a long and arduous task that requires strong and steady supporting measures to ensure the overall planning and implementation to approach perfection gradually.

\section{ACKNOWLEDGMENT}

This work is supported by Science and Technology Program of Gansu Province (1308RJZA214), the Young Scholars Science Foundation of Lanzhou Jiaotong University (2013032).

\section{REFERENCES}

[1] J. Zhang, Digital campus planning and implementation (2nd edition), Beijing: Electronic Industry Press, 2012..

[2] H. Wu and L. Qi, Exploring the construction of university Campus Data Center in the new era of information technology, http://info.cic.tsinghua.edu.cn/upload_file/_temp/1172827620798/ 1172827620798.pdf

[3] B. Christopher, A Brief Guide To Cloud Computing, Constable \& Robinson, 2010.

[4] P. Liu, Cloud Computing (2nd edition), Beijing: Electronic Industry Press, 2011.

[5] Campus Cloud Computing: to build an efficient campus, China Education Daily, http://www.chinacloud.cn/show.aspx $? \mathrm{id}=11087 \& \mathrm{cid}=16$

[6] C. Wang, VMware virtualization and Cloud Computing Application Cases, Beijing: China Railway Publishing, 2013.

[7] J. Huang, Development and construction of Cloud Computing in universities, China Education Network, 2012.

[8] F. Meng, M. Xu and W. Zhang, "Universities' Data Center design and implementation based on Cloud Computing", Modern Educational Technology, vol.22, 2012, pp. 99-103

[9] X. Meng, "Analysis of the data center building based on Cloud Computing”, China Enterprise Education, vol.22, 2012, pp.240241

[10] Z. Sun, "The requierments and realization method to build a universities' Data Center based on IaaS model", Technological Innovation and Application, col. 15, 2013, pp. 47-48 